\title{
Incidence of Dengue in Ozamiz City, Philippines
}

\author{
Jezreel Marc E. Pasay ${ }^{1}$, Anthony L. Awa ${ }^{2}$, Jesus G. Ocapan ${ }^{1}$ \\ ${ }^{1}$ College of Nursing and School of Midwifery, College of Arts and Sciences, Misamis \\ University, Ozamiz City, Philippines \\ ${ }^{2}$ College of Arts and Sciences, Misamis University, Ozamiz City, Philippines \\ Corresponding email: jmepasay14@gmail.com
}

\begin{abstract}
The incidence of dengue has become a serious health concern in many areas around the world. The increasing number of dengue cases are said to be the result of rapid population growth and climate change. This study assessed the incidence of dengue in Ozamiz City, Philippines. It also determined the relationship of climatic factors on dengue incidence reported in the area. Documentary analysis was done in seven hospitals to identify the incidence of dengue in the city. Pearson's correlation was used to determine the association between climatic factors and dengue incidence. Multiple regression analysis was also used as a statistical model. A total of 1,908 dengue cases were reported from January 2008 to September 2013. Majority of the patients belonged to the age group of 1-10 years. Maximum number of cases was seen in 2010 (398 cases) which was highest in the month of January (215 cases). Out of the total 1,908 cases, 821 $(43.03 \%)$ were dengue hemorrhagic fever, 781 (40.93\%) were dengue fever and 306 (16.04\%) were dengue shock syndrome. Among the 51 Barangays in the City, Barangay Aguada has the highest number of 186 cases $(9.75 \%)$. Of all climatic factors, only relative humidity has a positive correlation with dengue incidence. Vector control and education campaign needs should be assessed as these factors may significantly influence the occurrence of dengue in the city. Strategic plans and measures for dengue prevention need to be implemented targeting young population.
\end{abstract}

Keywords: Barangay Aguada, climate change, health, humidity, vector 


\section{Introduction}

Dengue is a viral infection transmitted primarily by the vector Aedes aegypti and Aedes albopictus mosquitoes. There are four known dengue serotypes that can cause severe and fatal disease; these are DENV-1, DENV-2, DENV-3 and DENV-4 (WHO, 2013a). The patient infected with a dengue virus experiences flu-like symptoms that lasts for two to seven days and may be accompanied by high fever $\left(40^{\circ} \mathrm{C}\right)$, headache, pain behind eyes, nausea and vomiting, swollen glands, joint, bone or muscle pains and rash. However, when developing into severe dengue cases such as dengue hemorrhagic fever and dengue shock syndrome, the patient can manifest a more complex and severe symptoms which are fatal to children (WHO, 2013b).

In the last three decades, outbreaks of dengue in many countries have increased ten-fold (Simmons \& Farrar, 2009). Highly urbanized countries like the America and even the middle-income countries in Asia are greatly affected. Dengue has become endemic in many tropical and subtropical countries around the world (Johansson et al., 2009). According to the World Health Organization in 2012, there are about 500,000 severe dengue cases reported annually with 12,500 deaths in the whole world in which $70 \%$ of the total global dengue morbidity and mortality cases occurs in the Southeast Asia region (Wu et al., 2011). Mounting evidences suggest that dengue expansion will continue unless a vaccine or antiviral drug is available.

Dengue incidence varies in all countries. In the current years, the Philippines recorded significant data of dengue cases. According to the Department of Health, National Epidemiology Center (2013), at least 42,207 dengue cases with 193 fatalities recorded from January up to the first week of June in 2013 which shows one percent decline or 456 fewer cases compared to the same period last year (42,663). A report from Department of Health, Regional Epidemiology (2013) showed that there were 2662 dengue cases across the region from January 1 to July 5, 2013. This is $32.83 \%$ higher than last year with 1,821 cases. Compared to other provinces in Northern Mindanao, Misamis Occidental has the highest increase of dengue incidence in terms of percentage $(381.94 \%)$ for this year. Among the three (3) cities in Misamis Occidental, Ozamiz City has the highest rise $(447 \%)$ of dengue cases. Despite the government's efforts against this disease, dengue fever still remains a major health problem. The reflected increase of dengue cases has been caused by many factors 
including human behavior, climate variability and global warming (Degallier et al., 2010). Global climate change might pose tremendous effects on dengue fever in the future (Hii et al., 2009). A study by Chowell and Sanchez (2006), found a correlation between temperature, precipitation or evaporation with the number of dengue cases. The possible relationship between dengue incidence and climatic factors encourages the use of information about climate for early detection and for establishing more effective prevention strategies (Dibo et al., 2008). However, preventive measures against dengue are not limited only about data on weather factors because climate change is not the sole culprit of dengue epidemics but from complex interactions between ecology, environment, vector and virus serotypes (Chandy et al., 2013).

Comprehensive information about the incidence of dengue and climatic influence of its occurrence is needed in formulating the more effective prevention measures. This study aimed to assess the incidence of dengue in Ozamiz City, Philippines. Also, this study determined the possible underlying correlation of climatic factors such as rainfall, minimum temperature, maximum temperature and humidity on dengue incidence from January 2008 to September 2013 using Pearson's correlation and multiple regression analysis.

\section{Materials and Methods}

Ozamiz City is located in the southern part of the province of Misamis Occidental and is nestled at the western side of the entrance of Panguil Bay in Region 10 in Northwestern Mindanao. The city consists of fifty one (51) barangays, twenty three (23) of these are urban and urbanizable, while twenty-eight (28) are rural. According to the 2010 census, it has a human population of 131,527. Average monthly temperature ranges from $22.7^{\circ} \mathrm{C}$ to $32.7^{\circ} \mathrm{C}$. Relative humidity ranges from $80 \%$ to $86 \%$. The average annual rainfall ranges from $1,415 \mathrm{~mm}$ to 3,758 $\mathrm{mm}$.

The total number of dengue cases was obtained from the official hospital logbooks of the following hospitals: MHARS Regional Training and Teaching Hospital, Medina General Hospital, Misamis University Medical Center, SM Lao Memorial City General Hospital, Faith Hospital, St. Joseph Hospital and Misamis Community Hospital. Dengue cases reported from January 2008 to September 2013 were gathered in a monthly basis. Monthly climatic data (rainfall, maximum temperature, 
minimum temperature and relative humidity) for Ozamiz City over the period of January 2008 to September 2013 was provided by Philippine Atmospheric, Geophysical and Astronomical Services (PAG-ASA) office in Cagayan de Oro City, Region X.

Pearson's correlation coefficient and multiple regression analysis were used to analyze the correlation between climatic factors and dengue incidence. Pearson's correlation measured the strength and direction of the linear relationship between climatic factors and dengue incidence. A +1 value of Pearson's correlation coefficient means that the two variables are perfectly linear related by an increasing relationship, a value of -1 shows that the variables are perfectly linear related by a decreasing relationship, and a value of 0 shows that the variables are not linear related by each other. There is a strong correlation if the correlation coefficient is greater than 0.8 and a weak correlation if the correlation coefficient is less than 0.5. Multiple Regression Analysis was also the statistical tool used to establish whether or not climatic factors are effective predictors of dengue incidence.

\section{Results and Discussion}

A total of 1,908 dengue cases were reported from January 2008September 2013 in seven hospitals in Ozamiz City. The highest number of dengue cases (398) was reported in the year 2010, whereas the lowest number was seen in 2012 with 166 dengue cases (Figure 1). There is also an increase of more than $100 \%$ dengue cases from the year 2012 to 2013. The uneven growth of dengue cases in the five-year period with increases seen in 2010 (398 cases), 2011 (394 cases) and 2013 (377 cases) are similar to the findings of the study in India (Sharma et al., 2012). 


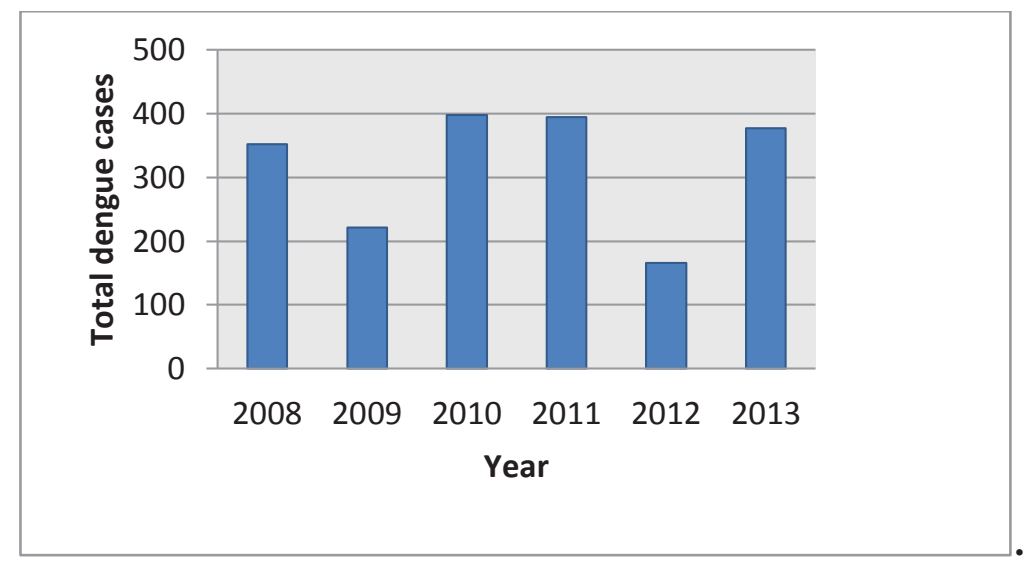

Figure 1. Reported dengue cases for January 2008 - September 2013.

Month-wise distribution of dengue cases (Figure 2) showed that most cases occurred in the month of January (215 cases) which was closely followed by March (211 cases). The month of November had the lowest number of dengue cases (98). Moreover, the data on the monthwise incidence of dengue showed that the number of dengue cases started to drop during the month of June up to the month of November. Dengue cases started to surge during the month of December to January. In this context, the month of December has one of the highest recorded rainfalls in the region. A study conducted in Thailand confirmed the effects of rainfall to the incidence of dengue infection (Wiwanitkit, 2006). Preventive measures against dengue infection should be instituted after bouts of rainfall. Water stagnation sources are rampant following heavy rainfall which could become favorable breeding sites for mosquitoes. Furthermore, unsteady growth of dengue cases is seen on figures 3 to 8 which show the monthly reports of dengue cases from January 2008 to September 2013. 


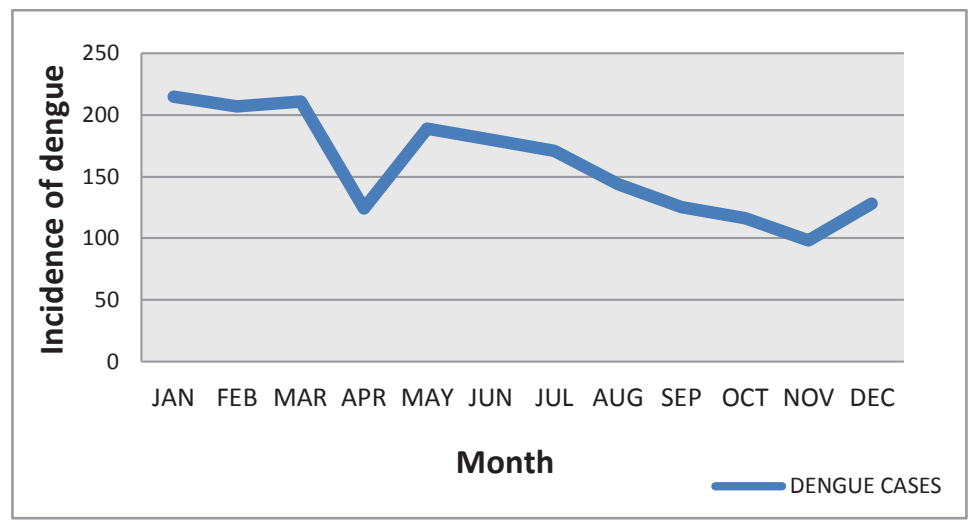

Figure 2. Average monthly reports of dengue cases for January 2008September 2013.

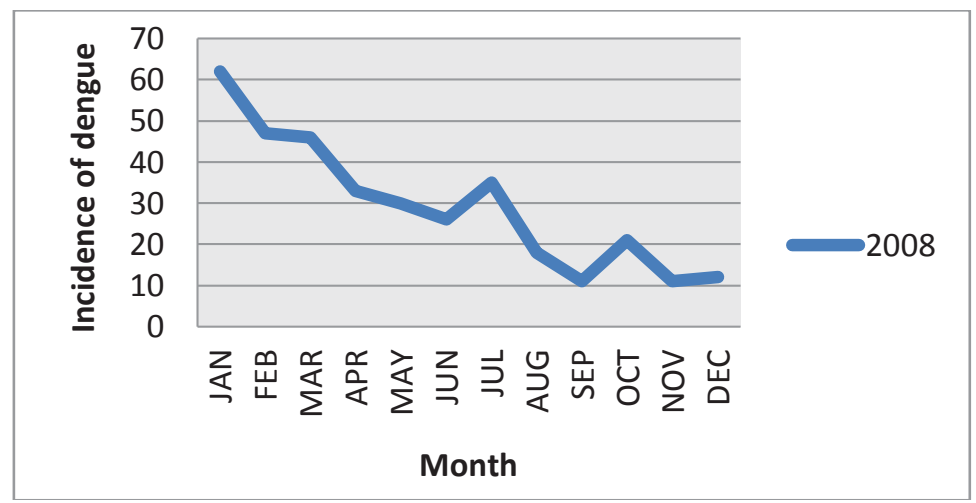

Figure 3. Monthly report of dengue cases for year 2008 .

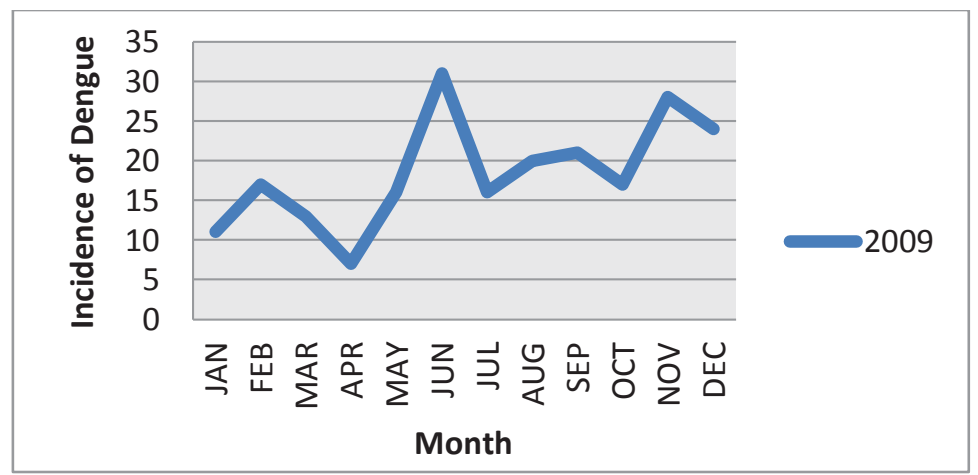

Figure 4. Monthly report of dengue cases for year 2009. 


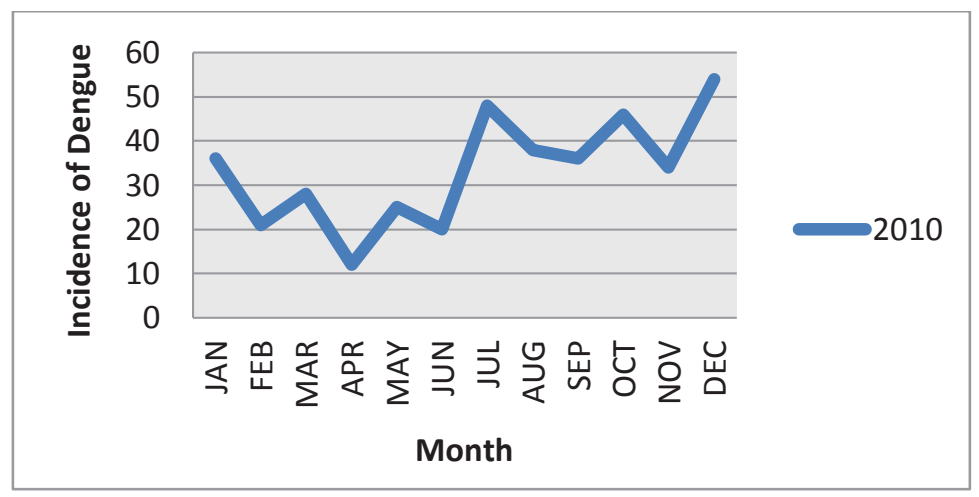

Figure 5. Monthly report of dengue cases for year 2010.

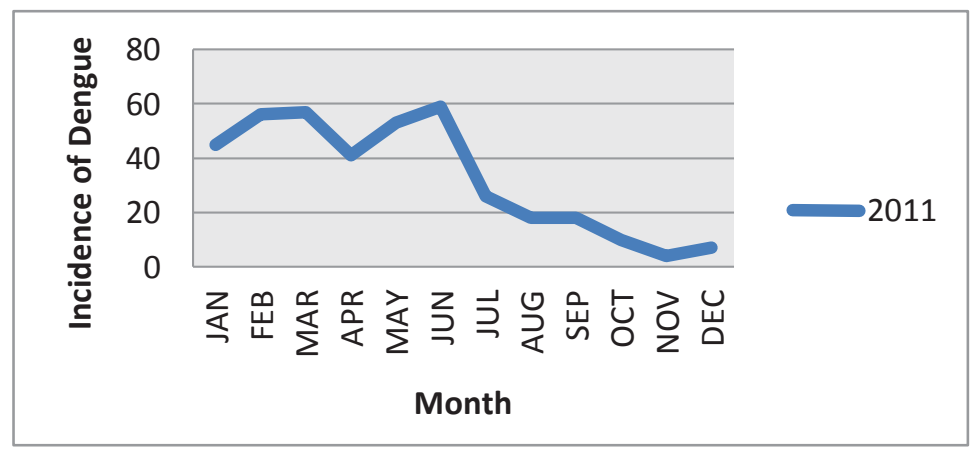

Figure 6. Monthly report of dengue cases for year 2011.

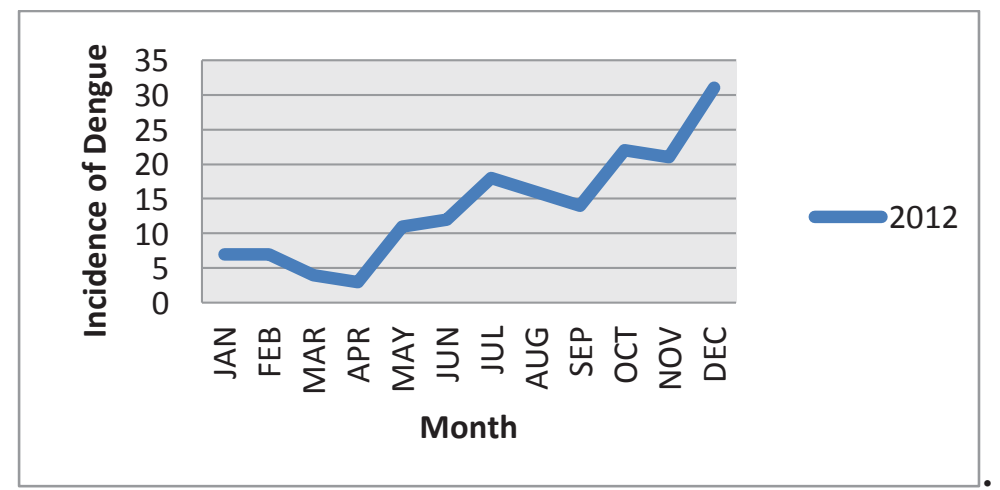

Figure 7. Monthly report of dengue cases for year 2012. 


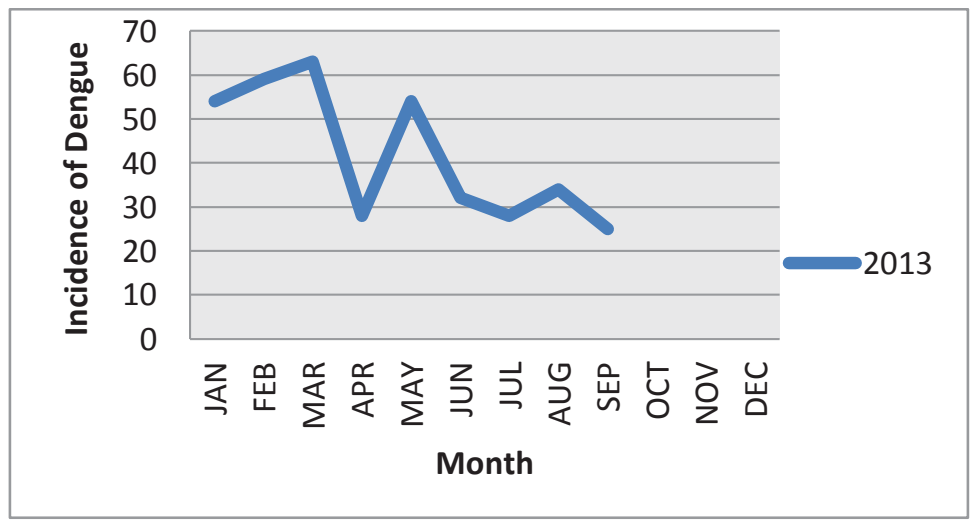

Figure 8. Monthly report of dengue cases for January-September 2013.

Dengue hemorrhagic fever is a life-threatening complication of dengue infection which is characterized by plasma leakage and hemorrhage. When shock occurs, the illness may lead to dengue shock syndrome which is more fatal. Out of the total 1,908 dengue cases confined to the different hospitals, 821 (43.03\%) were categorized as dengue hemorrhagic fever (DHF), 781 (40.93\%) were diagnosed as dengue fever and $306(16.04 \%)$ had dengue shock syndrome (DSS) (Figure 9). Notable differences in the monthly distribution of DHF and DSS from January 2008 to September 2013 are further shown in figures 10-15 with the highest incidence of dengue hemorrhagic fever recorded on the month of January 2008. The highest recorded case of dengue shock syndrome was on the month of June 2011. DHF and DSS are considered one of the major causes of morbidity and mortality among children in Southeast Asian countries (Kouri et al., 1989). Since mostly children suffered from these complications, mothers or guardians should have enough information about the signs and symptoms of DHF and DSS. Educational campaigns about the clinical manifestations of DHF and DSS are necessary for early detection and prompt treatment of complications. 


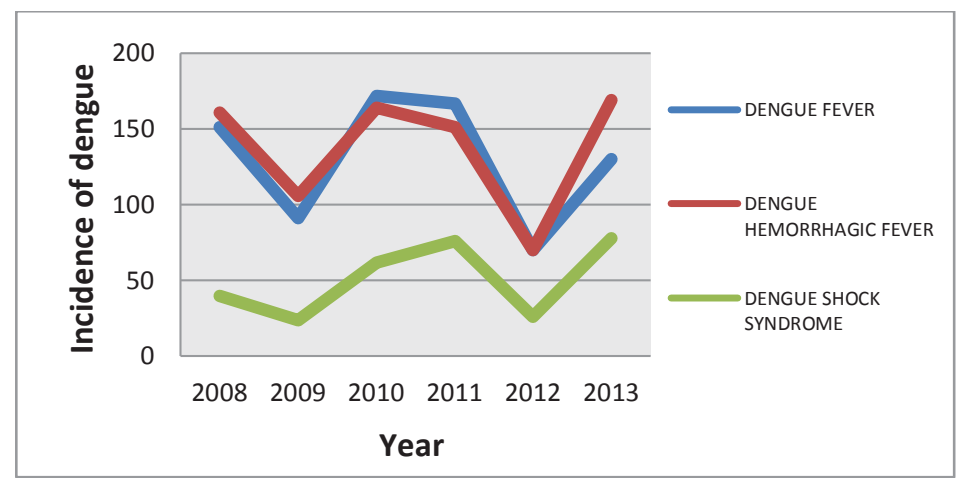

Figure 9. Yearly reports of DF, DHF, DSS cases for January 2008September 2013.

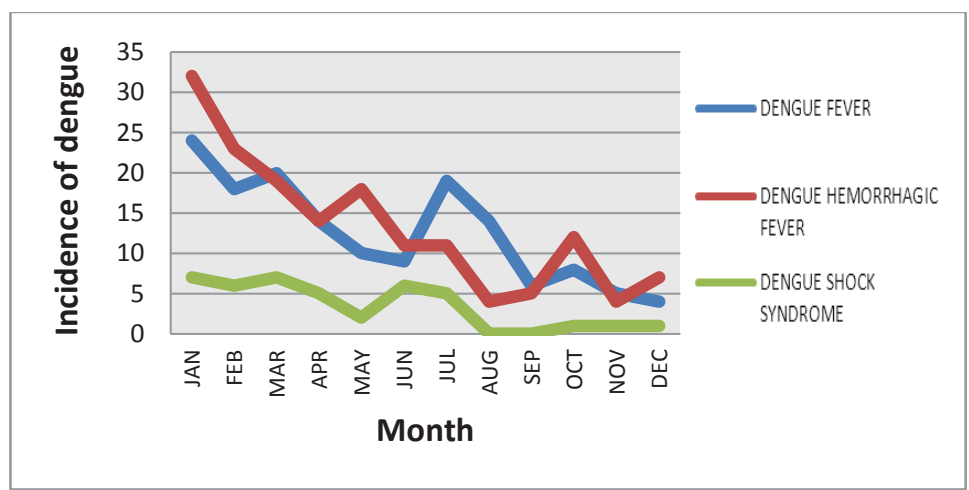

Figure 10. Reported cases of DF, DHF and DSS for year 2008.

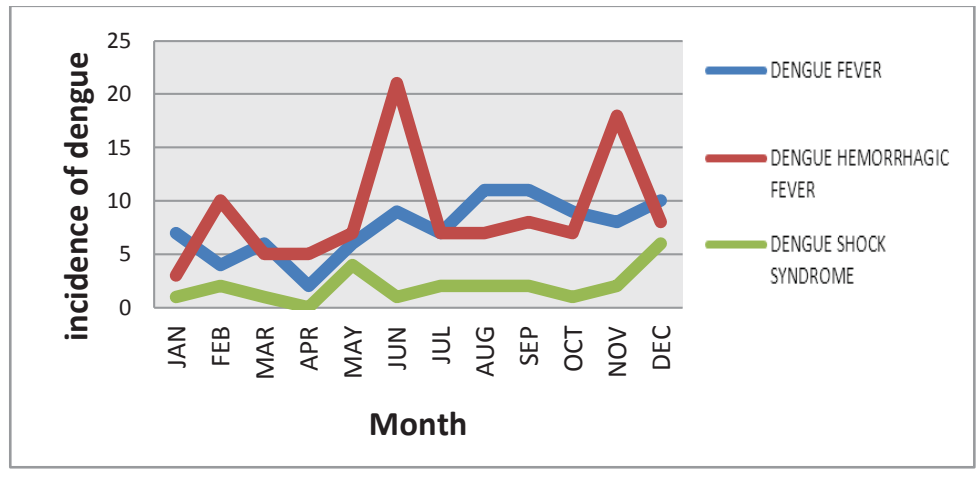

Figure 11. Reported cases of DF, DHF and DSS for year 2009. 


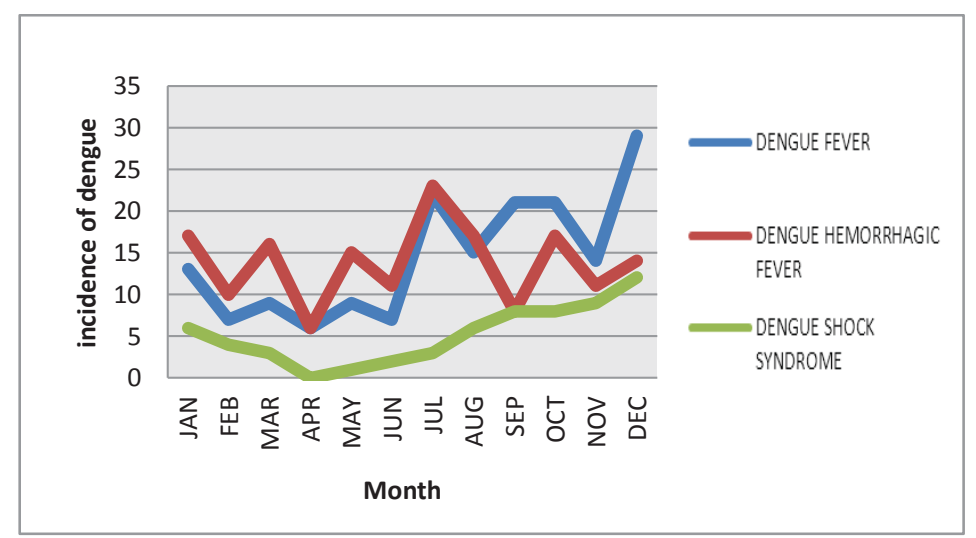

Figure 12. Reported cases of DF, DHS and DSS for year 2010

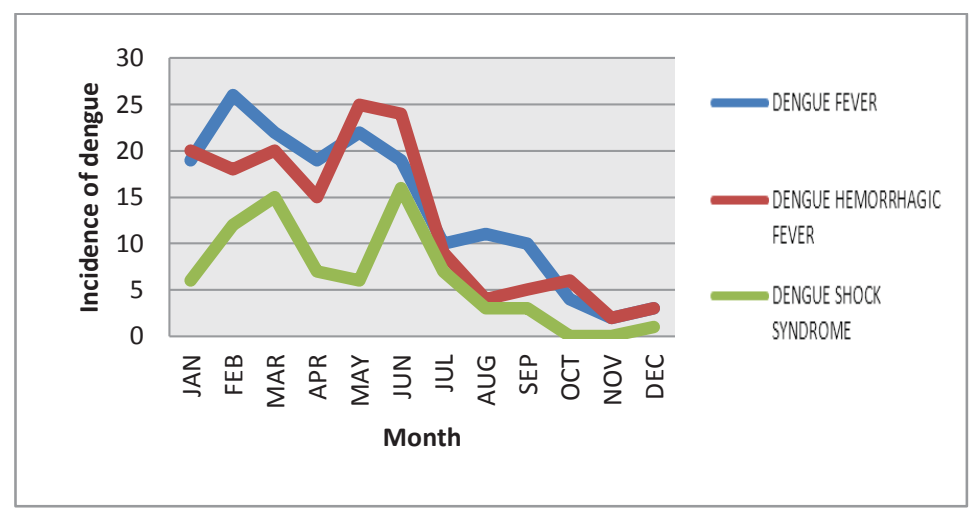

Figure 13. Reported cases of DF, DHF and DSS for year 2011

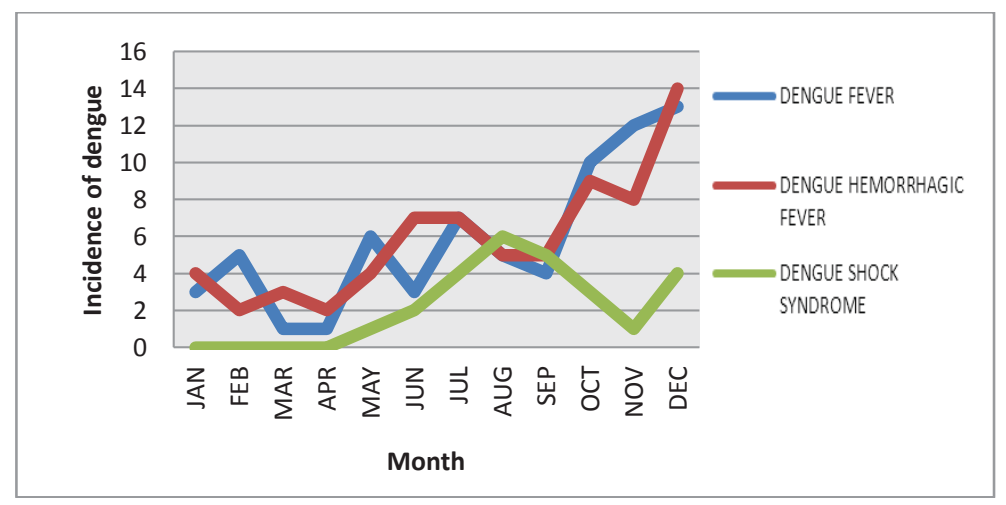

Figure 14. Reported cases of DF, DHF and DSS for year 2012 


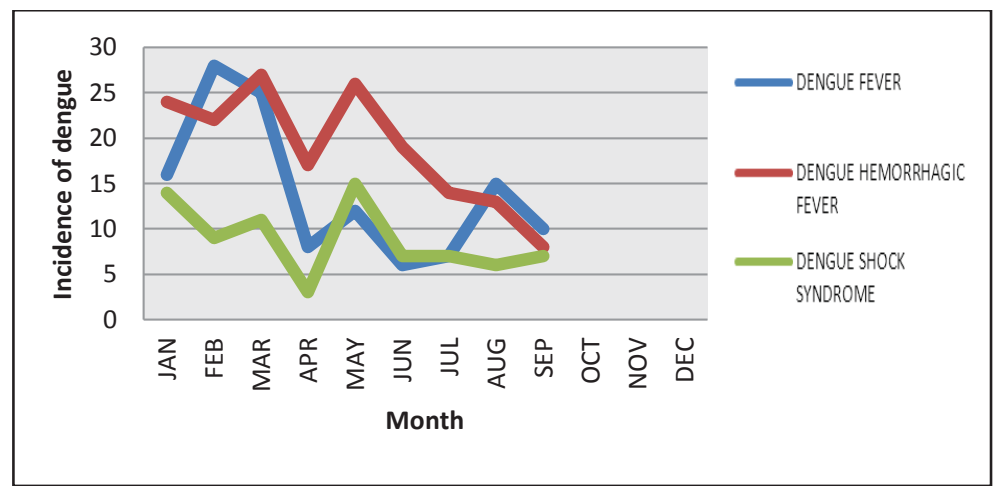

Figure 15. Reported cases of DF, DHF and DSS for January-September 2013.

Patients in the age group of 1-10 years old were commonly affected with dengue infection followed by those in the age group of 11-20 years old (Table 1). Studies in other countries showed wide variations of age distribution of dengue cases. The finding of this study is similar to the retrospective study in Thailand (Chareonsook et al., 1999). However, a study in Cambodia yielded different results in which infants aged $<1$ year old were mostly affected (Huy et al., 2010). In Puerto Rico, Nicaragua and India, the age group most commonly affected was 10-20 years (Rigau-Perez et al., 2001; Standish et al., 2010; Kumar et al., 2010). Dengue mainly affected adults in Brazil (Cordeiro et al., 2007). As multiple serotypes of dengue increases in a certain geographic area, adults have a lower probability to become susceptible to infection. For this reason, young population becomes more prone individuals to acquire dengue infection (Sharma et al., 2012). The trend from this increased incidence among young population has important implications for control and prevention of dengue diseases (Guha-Sapir \& Schimmer, 2005). Information and educational campaigns about dengue prevention and control should also be extended to schools where large population belongs to this age group commonly affected with dengue infection. 
Table 1. Age-wise distribution of dengue cases (2008-2013).

\begin{tabular}{cc}
\hline Age & Dengue cases \\
\hline & \\
$1-10$ & 47 \\
$11-20$ & 758 \\
$21-30$ & 612 \\
$31-40$ & 246 \\
$41-50$ & 104 \\
$51-60$ & 53 \\
$61-70$ & 42 \\
$71-80$ & 28 \\
$81-90$ & 13 \\
Total & 5 \\
\hline
\end{tabular}

Mosquitoes that cause dengue infection are regarded as "urban" mosquitoes because of their adaptation to survive among humans and continually produce their eggs in standing water around houses (Gubler \& Meltzer, 1999). In this study, dengue patients admitted in hospitals came from 49 Barangays in Ozamiz City out of 51 Barangays (Figure 16). Most of the patients came from urban barangays such as Barangay Aguada with the highest number of constituents affected with 186 cases $(9.75 \%)$ and Barangay Tinago with 146 cases (7.65\%). Barangays with the lowest cases are Manaka, Liposong and Guingona with one case each. Figures 17-22 show that dengue infection has greatly expanded geographically in the area every year. Highest incidence of dengue in a single locality is recorded in Barangay Aguada with 49 cases during the period of January to September 2013. The findings further showed that dengue cases did not only occur in urban areas but also in neighboring rural barangays. In this connection, vector control campaigns must not only confine in urban areas but in rural areas as well. 


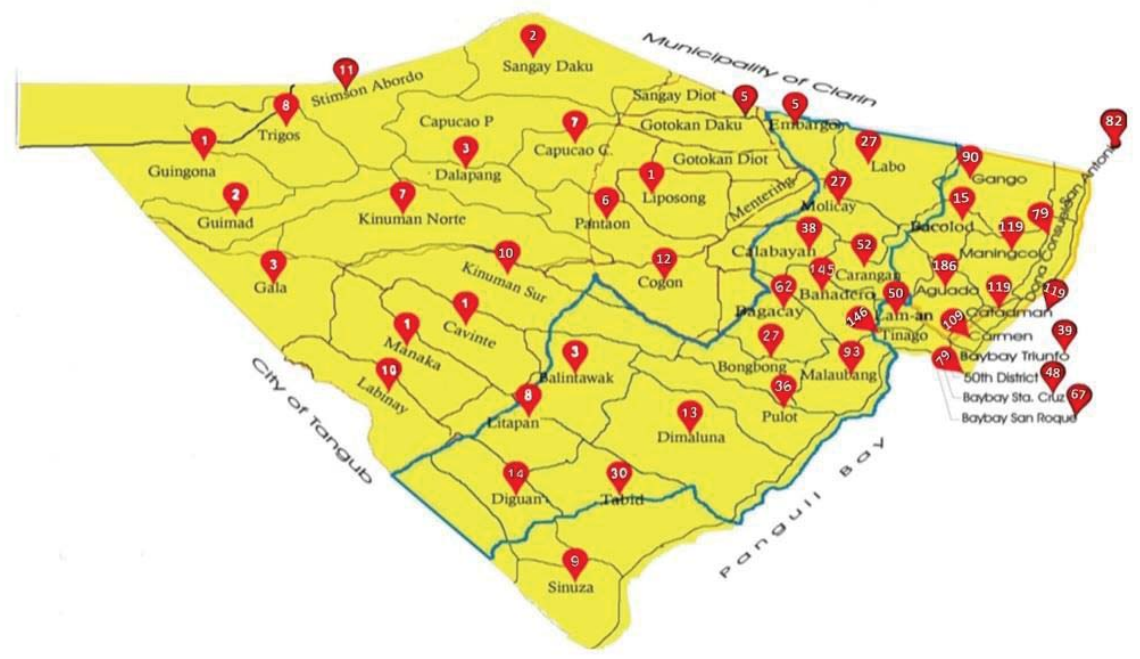

Figure 16. Barangay-wise distribution of dengue cases for January 2008September 2013 (Number in red balloon pointing to a particular locality represents the number of dengue cases affecting the Barangay).

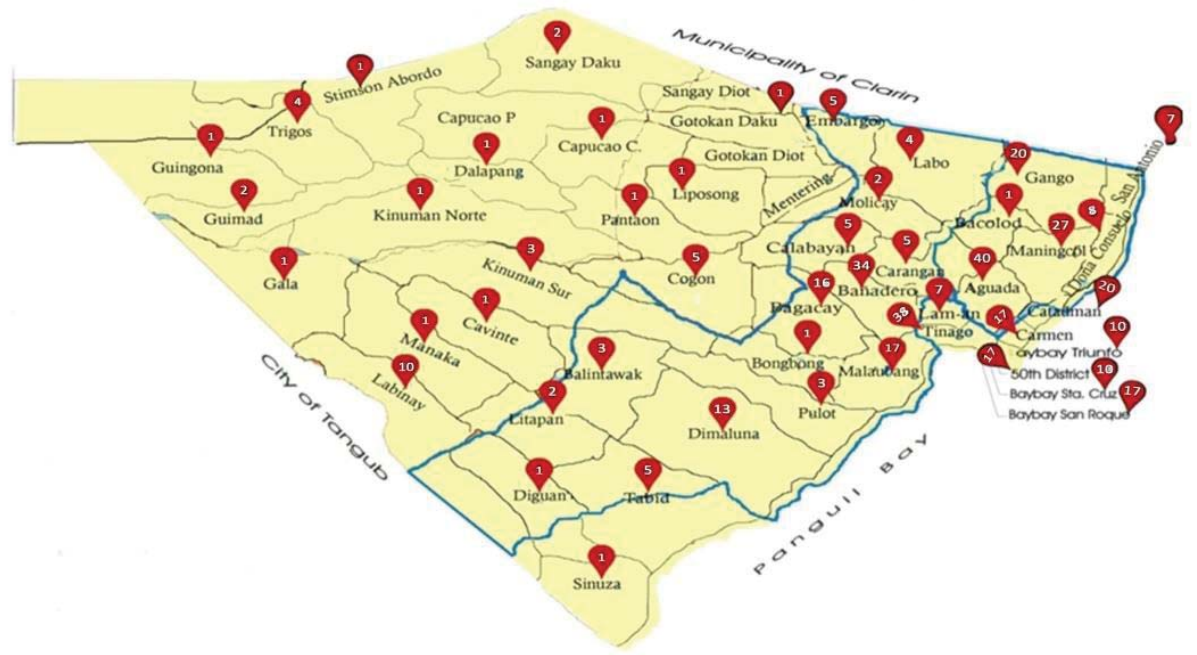

Figure 17. Barangay-wise distribution of dengue cases for year 2008 (Number in red balloon pointing to a particular locality represents the number of dengue cases affecting the Barangay. 


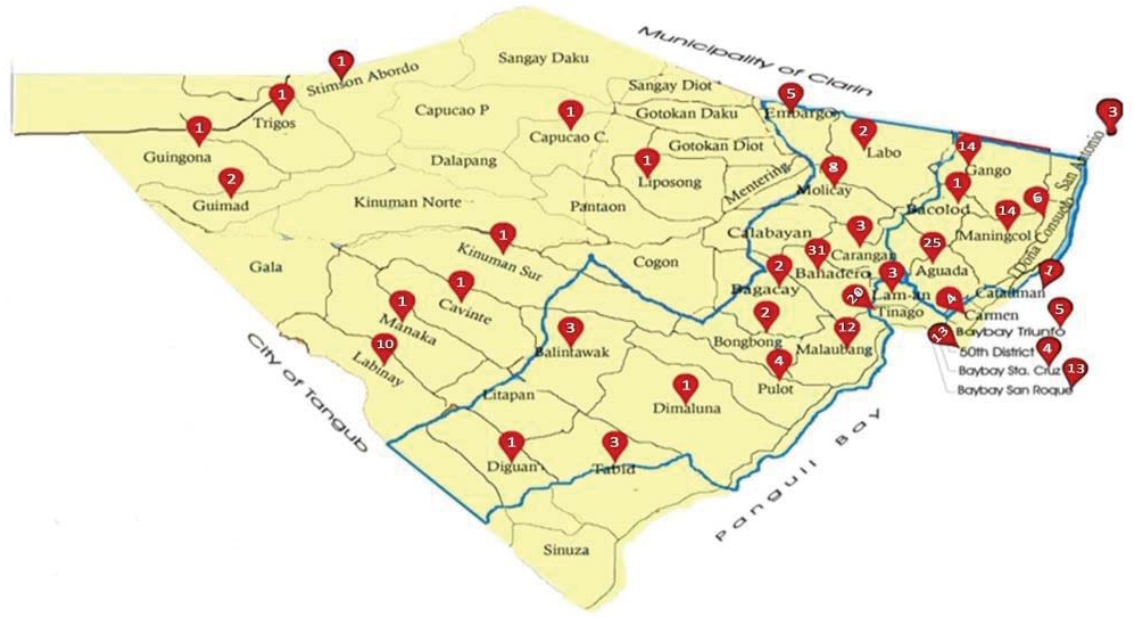

Figure 18. Barangay-wise distribution of dengue cases for year 2009 (Number in red balloon pointing to a particular locality represents the number of dengue cases affecting the Barangay).

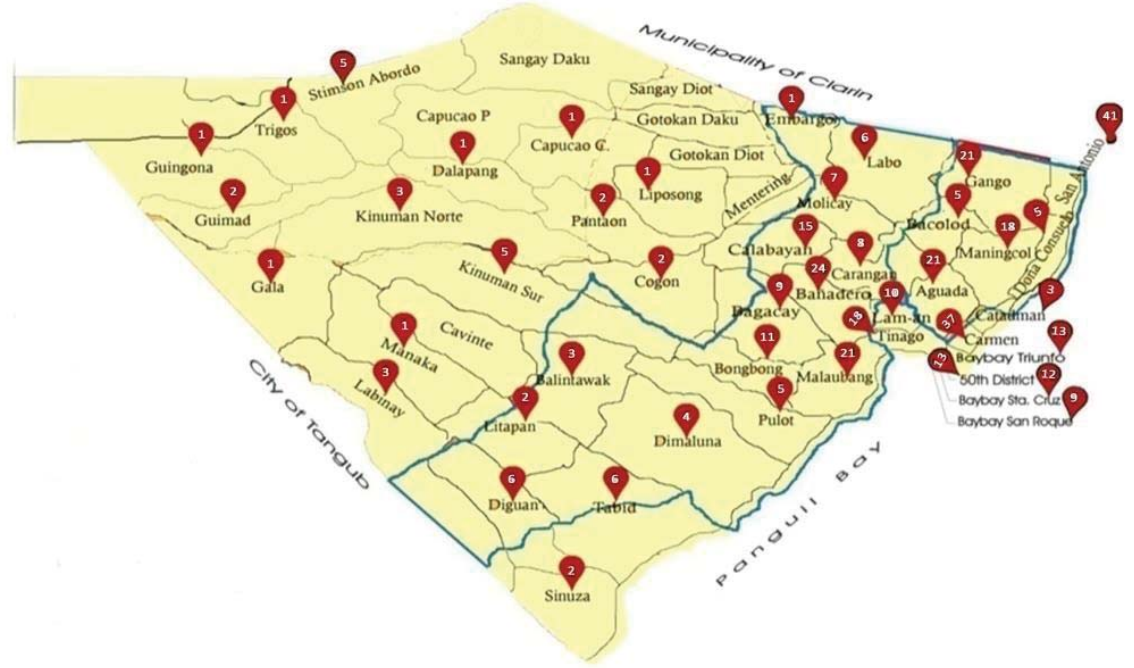

Figure 19. Barangay-wise distribution of dengue cases for year 2010 (Number in red balloon pointing to a particular locality represents the number of dengue cases affecting the Barangay) 


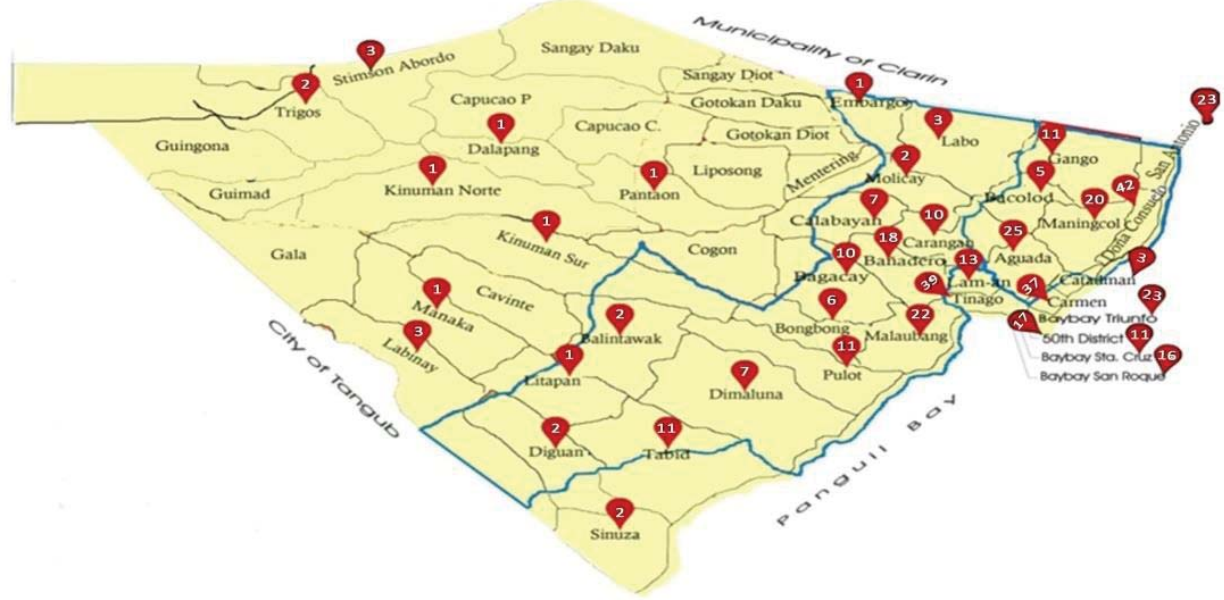

Figure 20. Barangay-wise distribution of dengue cases for year 2011 (Number in red balloon pointing to a particular locality represents the number of dengue cases affecting the Barangay).

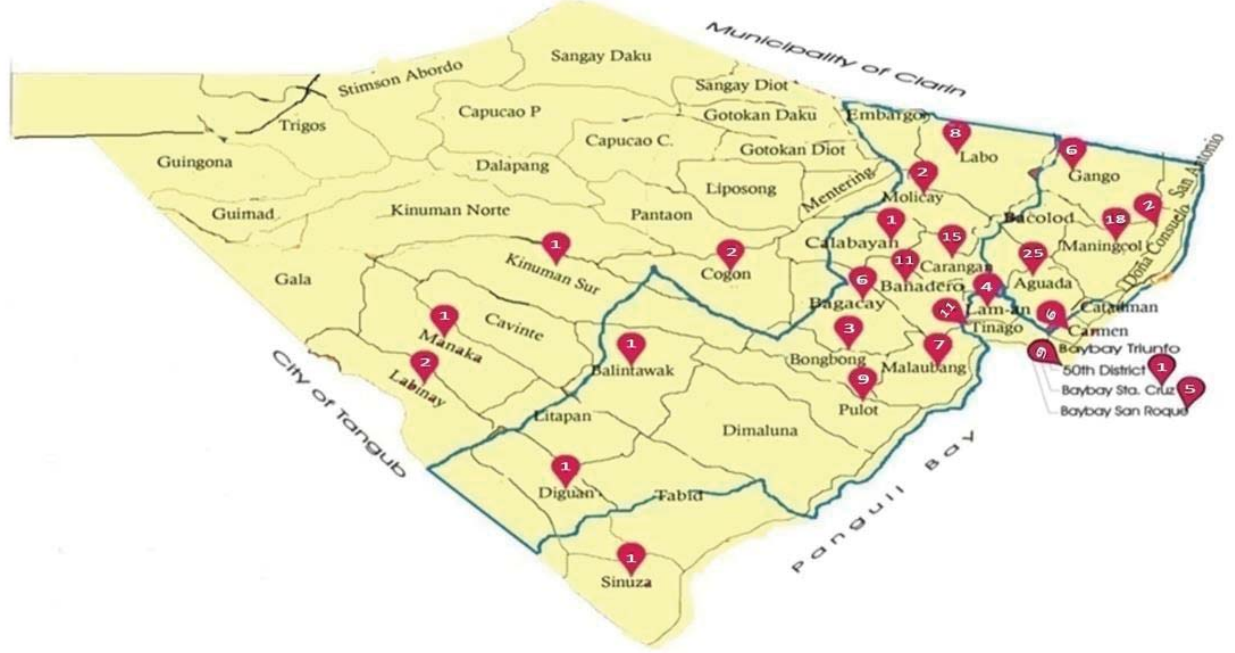

Figure 21. Barangay-wise distribution of dengue cases for year 2012 (Number in red balloon pointing to a particular locality represents the number of dengue cases affecting the Barangay). 


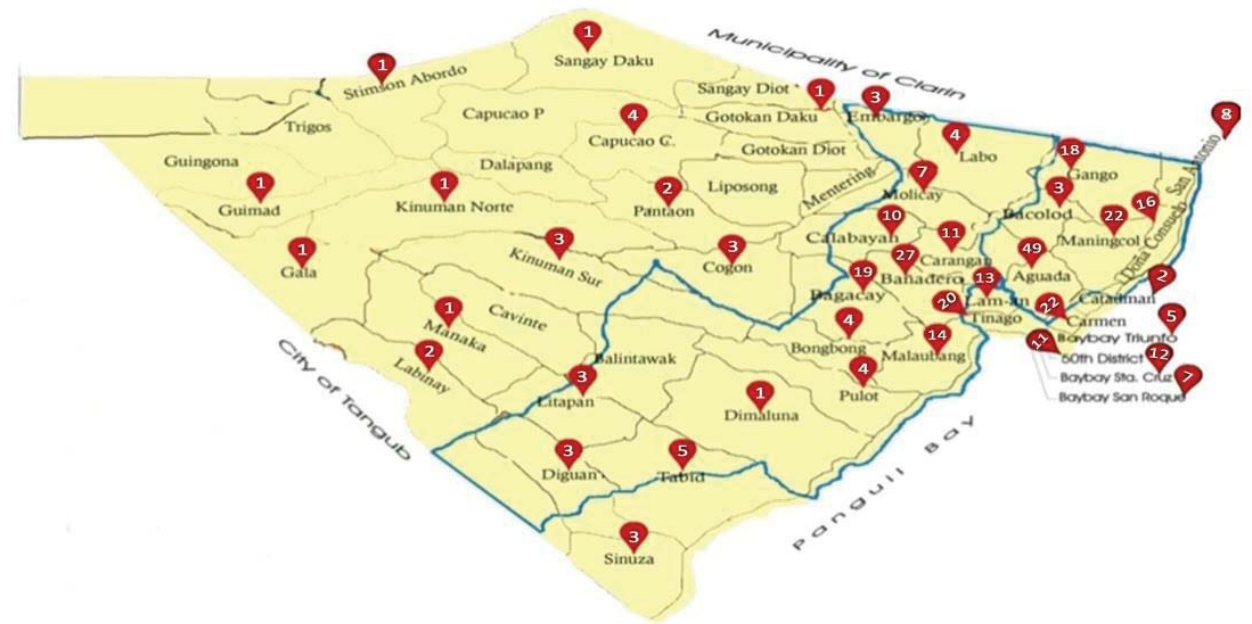

Figure 22. Barangay-wise distribution of dengue cases for year 2013 (Number in red balloon pointing to a particular locality represents the number of dengue cases affecting the barangay).

This study also looked into the relationship of the incidence of dengue infection with climatic factors such as rainfall, temperature and humidity. The month of May signifies the start of the rainy season (Figure 23). Average rainfall continues to increase until the month of June $(210.05 \mathrm{~mm})$ until it reaches its highest point in the month of July $(271.07 \mathrm{~mm})$. On the other hand, the month of April is the driest month with an average rainfall of $63.60 \mathrm{~mm}$. There is no definitive trend between rainfall and incidence of dengue. However, a noted decrease in dengue incidence in the months of March and April corresponds to the decrease in values of rainfall on the same months, with an observed increase on the following month of both variables. Climatic factor like rainfall was linked with dengue incidence in a study by Sia Su (2008) in Metro Manila, Philippines. 


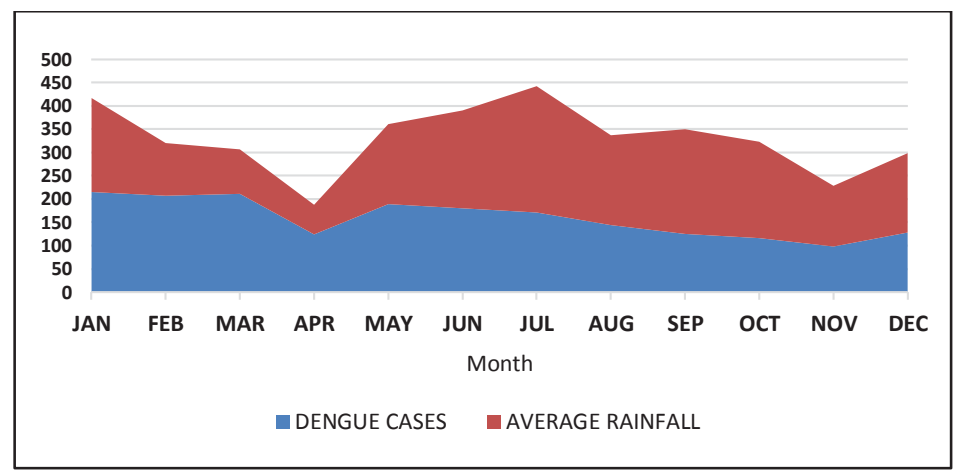

Figure 23. Dengue cases in relation to rainfall (January 2008 - September 2013).

The month of May was recorded as the hottest month over a five-year period with an average maximum temperature of $33.05^{\circ} \mathrm{C}$ while February is the coldest month with a minimum temperature of $21.92^{\circ} \mathrm{C}$ (Figure 24). According to Alexander et al. (2006), there is a widespread change in temperature associated with warming over $70 \%$ of global land throughout the $20^{\text {th }}$ century. This likely supports the climate change in the region. Per observation, the highest incidence of dengue in the month of January coincides with the lowest value of maximum temperature. Temperature played an important role in dengue incidence in Thailand (Promprou et al., 2005).

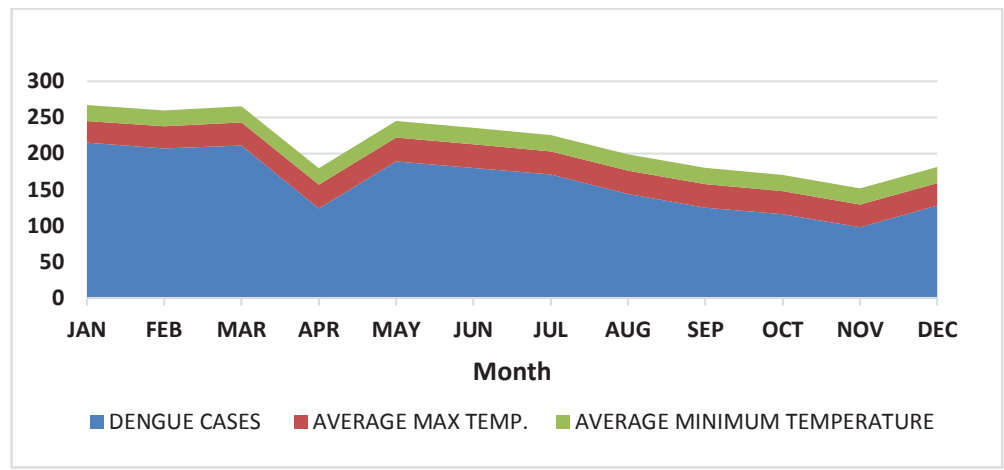

Figure 24. Dengue cases in relation to maximum and minimum temperature (January 2008-September 2013). 
The month with the highest recorded humidity $(85.50 \%)$ in 5 years is January (Figure 25). There is a slight decrease $(84.67 \%)$ on the month of February and a consistent decline (82.00\%) was shown during the month of March until it continues to fall to its lowest level $(79.33 \%)$ in the month of April. Based on recorded data, the highest recorded humidity level in January coincides with the highest cases of dengue fever in January. A study in Brazil and Taiwan found an association of dengue incidence with relative humidity (Goncalves \& Rebelo, 2004; Wu et al., 2011).

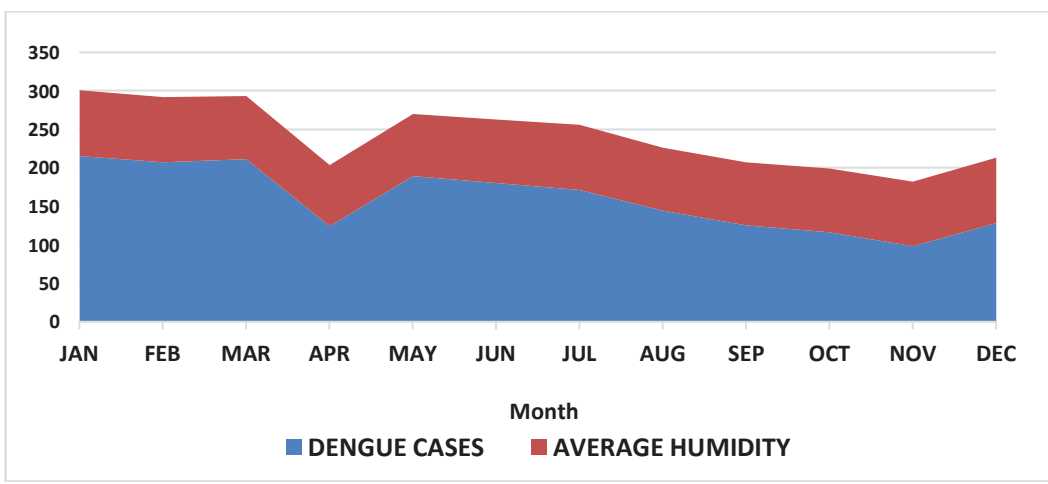

Figure 25. Dengue Cases in Relation to Humidity January 2008-September 2013.

Table 2 shows the Pearson's correlation coefficient analysis between dengue incidence and climatic factors. Results revealed that climatic factors such as rainfall, maximum and minimum temperature acquired negative values of Pearson correlation coefficient (-.04, -.277 and -.205 respectively). This indicates inverse and weak linear relationship. Therefore, an increase in rainfall, maximum and minimum temperature has a little corresponding decrease in dengue cases. On the other hand, relative humidity had a positive but weak correlation coefficient (.223). This means that an increase or decrease on the values of relative humidity has direct or indirect effect on the number of dengue cases. Results of multiple regression analysis showed insignificant correlation between the dengue incidence and the climatic factors (Table 3). It means that climatic factors are not effective predictors of dengue incidence. Accordingly, other factors may likely influence the incidence of dengue in the area such as control of vector population and efforts to educate people on how to avoid dengue infections. Immunity of the population to infection, presence 
of new serotypes and demographic factors could have influenced the transmission of dengue in the area (Clarke, 2002). Based on the results of Pearson's correlation coefficient analysis, the only climatic factor that has a positive correlation with dengue incidence is humidity. Moisture was the most significant predictor of dengue occurrence (Hales et al., 2002). According to Dulay et al. (2013), relative humidity influences longevity, mating, dispersal, feeding behavior and oviposition of mosquitoes and rapid replication of the virus. Hales et al. (2002) added that high relative humidity increases the survival and breeding conditions of mosquitoes.

Table 2. Correlation between dengue incidence and climatic factors.

\begin{tabular}{cccccc}
\hline \hline & & $\begin{array}{c}\text { Average } \\
\text { Rainfall }\end{array}$ & $\begin{array}{c}\text { Temp } \\
\text { (Max.) }\end{array}$ & $\begin{array}{c}\text { Temp } \\
\text { (Min.) }\end{array}$ & $\begin{array}{c}\text { Relative } \\
\text { Humidity }\end{array}$ \\
\hline \multirow{2}{*}{ Dengue } & Pearson & -.014 & -.277 & -.205 & .223 \\
Incidence & Correlation & & & & .522 \\
& Sig (2-tailed) & .996 & .384 & .486 \\
& $\mathrm{~N}$ & 12 & 12 & 12 & 12 \\
\hline \hline
\end{tabular}

*correlation is significant at 0.05 level

Table 3: Relationship between climatic factors with dengue incidence

\begin{tabular}{cccccc}
\hline \hline & & $\begin{array}{c}\text { Average } \\
\text { Rainfall }\end{array}$ & $\begin{array}{c}\text { Temp } \\
\text { (Max.) }\end{array}$ & $\begin{array}{c}\text { Temp } \\
\text { (Min.) }\end{array}$ & $\begin{array}{c}\text { Relative } \\
\text { Humidity }\end{array}$ \\
\hline \multirow{2}{*}{$\begin{array}{c}\text { Dengue } \\
\text { Incidence }\end{array}$} & $\begin{array}{c}\text { Standard } \\
\text { Coefficient }\end{array}$ & -.034 & -.525 & .179 & -.103 \\
& $\mathrm{~T}$ & -.056 & -.428 & .216 & -.095 \\
& Sig. & .957 & .682 & .835 & .927 \\
\hline \hline
\end{tabular}

\section{Conclusion and Recommendations}

A total of 1,908 dengue cases were reported from January 2008September 2013 in seven hospitals in Ozamiz City. The highest number of dengue cases (398) was reported in the year 2010, whereas the lowest number was seen in 2012 with 166 dengue cases. Month-wise distribution of dengue cases has shown that the month of January has the most cases. The age group of 1-10 years was mostly affected with the infection. There were 821 dengue hemorrhagic fever cases, 781 dengue fever cases and 306 cases of dengue shock syndrome. Among the 51 barangays covered in Ozamiz City, barangay Aguada has the highest incidence with 186 dengue cases followed by barangay Tinago with 146 cases. Though the majority 
of the cases came from urban barangays, neighboring rural areas in the city were also affected. Results from Pearson's correlation coefficient analysis showed that there is a positive but weak linear relationship between relative humidity and dengue incidence. An increase or decrease in relative humidity has a little effect on the incidence of dengue cases. On the other hand, rainfall, maximum and minimum temperature has inverse and weak linear relationship with dengue incidence. Therefore, an increase in rainfall, maximum and minimum temperature has little corresponding decrease in dengue cases. Climatic factors showed insignificant correlation with dengue incidence in this study based on the multiple regression analysis.

Other factors should be assessed as predictors to dengue incidence in Ozamiz City. Intensive vector control should be implemented in connection with the increased dengue incidence from 2012 to 2013. It can be achieved through an integrated approach with the combination of environmental management, chemical control and biological methods. This study is the first in this area to report dengue cases per barangay and specifically identify the susceptible age group. The findings of this study may be used to plan out and implement different strategies and measures targeting the young population.

\section{Acknowledgment}

The authors acknowledge the cooperation extended by the staff in all hospitals covered by the study. More importantly, the authors gratefully acknowledge Misamis University for the research grant.

\section{Literature Cited}

Alexander, L. V., Zhang, X., Peterson, T. C., Caesar, J., Gleason, B., Klein Tank, A. M. G., ... \& Vazquez-Aguirre, J. L. (2006). Global observed changes in daily climate extremes of temperature and precipitation. Journal of Geophysical Research: Atmospheres (19842012), 111(D5).

Chandy, S., Ramanathan, K., Manoharan, A., Mathai, D., \& Baruah, K. (2013). Assessing effect of climate on the incidence of dengue in Tamil Nadu. Indian journal of medical microbiology, 31(3). 
Chareonsook, O., Foy, H. M., Teeraratkul, A., \& Silarug, N. (1999). Changing epidemiology of dengue hemorrhagic fever in Thailand. Epidemiology and infection, 122(1), 161-166.

Chowell, G., \& Sanchez, F. (2006). Climate-based descriptive models of dengue fever: The 2002 epidemic in Colima, Mexico. Journal of environmental health, 68(10), 40.

Clarke, T. (2002). Dengue virus: break-bone fever. Nature, 416(6882), 672-674.

Cordeiro, M. T., Schatzmayr, H. G., Nogueira, R. M. R., Oliveira, V. F. D., Melo, W. T. D., \& Carvalho, E. F. D. (2007). Dengue and dengue hemorrhagic fever in the State of Pernambuco, 1995-2006. Revista da Sociedade Brasileira de Medicina Tropical, 40(6), 605-611.

Degallier, N., Favier, C., Menkes, C., Lengaigne, M., Ramalho, W. M., Souza, R., ... \& Boulanger, J. P. (2010). Toward an early warning system for dengue prevention: Modeling climate impact on dengue transmission. Climatic Change, 98(3-4), 581-592.

Department of Health, Regional Epidemiology. (2013). Surveillance and Disaster Response Unit Center for Health Development-Northern Mindanao. Retrieved from www.doh.gov.ph

Department of Health, National Epidemiology Center. (2013). Disease Surveillance. Retrieved from www.doh.gov.ph/diseasesurveillance.html

Dibo, M. R., Chierotti, A. P., Ferrari, M. S., Mendonça, A. L., \& Chiaravalloti Neto, F. (2008). Study of the relationship between Aedes (Stegomyia) aegypti egg and adult densities, dengue fever and climate in Mirassol, state of São Paulo, Brazil. Memórias do Instituto Oswaldo Cruz, 103(6), 554-560.

Dulay, A. V. S., Bautista, J. R., \& Teves, F. G. (2013). Climate Change and Incidence of Dengue Fever (DF) and Dengue Hemorrhagic Fever (DHF) in Iligan City, Lanao del Norte, Philippines. International Research Journal of Biological Sciences, 2(7), 37-4. 
Goncalves, N. \& Rebelo, J. M. (2004). Epidemiological characteristics of dengue in the Municipality of Sao Luis, Maranhao, Brazil 1997-2002. Cad Saude Publica, 20, 1424-31.

Guha-Sapir, D., \& Schimmer, B. (2005). Dengue fever: new paradigms for a changing epidemiology. Emerg Themes Epidemiol, 2(1), 1-10.

Gubler, D. J., \& Meltzer, M. (1999). Impact of dengue/dengue hemorrhagic fever on the developing world. Advances in Virus Research, 53, 35-70.

Hales, S., de Wet, N., Maindonald, J., \& Woodward, A. (2002). Potential effect of population and climate changes on global distribution of dengue fever: an empirical model. The Lancet, 360(9336), 830-834.

Hii, Y. L., Rocklöv, J., Ng, N., Tang, C. S., Pang, F. Y., \& Sauerborn, R. (2009). Climate variability and increase in intensity and magnitude of dengue incidence in Singapore. Global Health Action,2. doi: 10.3402/gha.v2i0.2036

Huy, R., Buchy, P., Conan, A., Ngan, C., Ong, S., Ali, R., ... \&Vong, S. (2010). National dengue surveillance in Cambodia 1980-2008: epidemiological and virological trends and the impact of vector control. Bulletin of the World Health Organization, 88(9), 650-657.

Johansson, M. A., Cummings, D. A., \& Glass, G. E. (2009). Multiyear climate variability and dengue-El Nino southern oscillation, weather, and dengue incidence in Puerto Rico, Mexico, and Thailand: a longitudinal data analysis. PLoS medicine, 6(11), e1000168.

Kouri, G. P., Guzmán, M. I. G., Bravo, J. R., \& Triana, C. (1989). Dengue haemorrhagic fever/dengue shock syndrome: Lessons from the Cuban epidemic, 1981. Bulletin of the World Health Organization, 67(4), 375. 
Kumar, A., Rao, C. R., Pandit, V., Shetty, S., Bammigatti, C., \& Samarasinghe, C. M. (2010). Clinical manifestations and trend of dengue cases admitted in a tertiary care hospital, Udupi district, Karnataka. Indian Journal of Community Medicine: Official Publication of Indian Association of Preventive \& Social Medicine, 35(3), 386.

Promprou, S., Jaroensutasinee, M., \& Jaroensutasinee, K. (2005). Climatic factors affecting dengue haemorrhagic fever incidence in Southern Thailand. Dengue Bulletin, 29, 41.

Rigau-Perez, J. G., Vorndam, A. V., \& Clark, G. G. (2001). The dengue and dengue hemorrhagic fever epidemic in Puerto Rico, 19941995. American Journal of Tropical Medicine and Hygiene, 64(1/2), 67-74.

Sharma, Y., Kaur, M., Singh, S., Pant, L., Kudesia, M., \& Jain, S. (2012). Seroprevalence and trend of dengue cases admitted to a government hospital, Delhi-5-year study (2006-2010): A look into the age shift. International journal of preventive medicine, 3(8), 537.

Simmons, C. P., \& Farrar, J. (2009). Changing patterns of dengue epidemiology and implications for clinical management and vaccines. PLoS Medicine, 6(9), e1000129.

Standish, K., Kuan, G., Avilés, W., Balmaseda, A., \& Harris, E. (2010). High dengue case capture rate in four years of a cohort study in Nicaragua compared to national surveillance data. PLoS Neglected Tropical Diseases, 4(3), e633.

Sia Su, G. L. (2008). Correlation of climatic factors and dengue incidence in Metro Manila, Philippines. AMBIO: A Journal of the Human Environment, 37(4), 292-294.

WHO. (2012). Dengue and Dengue Hemorrhagic Fever. Fact sheet 117. World Health Organization. Retrieved from http://www.who.int/ mediacentre/factsheets/fs117/en 
WHO. (2013a). Health Topics-Dengue. Retrieved from http://www.who. int/topics/dengue/en

WHO. (2013b). Programs and Projects-Dengue Control-Dengue/Severe Dengue. Retrieved from http://www.who.int/denguecontrol/faq/en/ index 1.html

Wiwanitkit, V. (2006). An observation on correlation between rainfall and the prevalence of clinical cases of dengue in Thailand. Journal of vector borne diseases, 43(2), 73.

Wu, F., Liu, Q., Lu, L., Wang, J., Song, X., \& Ren, D. (2011). Distribution of Aedes albopictus (Diptera: Culicidae) in northwestern China. Vector-Borne and Zoonotic Diseases, 11(8), 1181-1186. 